\title{
Micromachined silicon cantilevers and tips for bidirectional force microscopy
}

\author{
R.A. Buser, J. Brugger and N.F. de Rooij \\ Institute of Microtechnology, University of Neuchâtel, Breguet 2, CH-2000 Neuchâtel, Switzerland \\ Received 12 August 1991
}

\begin{abstract}
A monocrystalline silicon lever with an integrated silicon tip for a force/friction microscope was realized. Theoretical studies have been carried out to find the shape and dimensioning according to the mechanical system requirements. Moreover, sharp tips with a high aspect ratio could be demonstrated.
\end{abstract}

\section{Introduction}

Scanning tunneling microscopes (STMs) and atomic force microscopes (AFMs), known under the expression scanning probe microscope (SPM), are well established methods to analyze surfaces, and the principle of their operation is published widely [1-3]. In AFM a point probe reacts mechanically on interatomic forces of the sample and generates a topographic view of the surface with an atomic resolution. The mechanical part typically consists of a cantilever as a spring element with a sharp tip at its end.

In the field of solid-state sensors and actuators, micromachining techniques were developed to become the appropriate tool for cantilever and tip fabrication [4]. Silicon dioxide $\left(\mathrm{SiO}_{2}\right)$ as well as silicon nitride $\left(\mathrm{Si}_{3} \mathrm{~N}_{4}\right)$ cantilevers have been reported [5]. Recently also a polysilicon cantilever was presented [6] using a sacrificial-layer technique.

In microelectronics itself such STM/AFM are employed as profilers. Thereby the chip surface is measured in three dimensions, which allows in the comparison with an ideal chip a control of quality and even the localization of defects. For this application it would be very important to have arrays in order to reduce the scanning time, which is only possible with batch-fabricated heads.
Similar requirements hold for writing with SPM and maybe also for the observation of dynamic processes.

The advantage of using thin films to form cantilevers for STM/AFM is the possible high aspect ratio to obtain a high stiffness in $x$ and $y$ direction (in the plane) and an adjustable stiffness in $z$ direction (out of the plane), without affecting the resonance frequency. We have fabricated such $\mathrm{SiO}_{2}$ cantilevers [4] which have been successfully used in AFM applications [3].

Recently, some interest has arisen to measure not only the force in the $z$ direction but also parallel to the scanning direction (say $y$ direction) especially to study tribological behaviour of surfaces. The effect of this force at the tip is either pushing the cantilever sideways or, when this one is too still in this direction, twisting it. Both possibilities have been applied for friction measurements. For the torsional type, micromachined cantilevers have been used $[7,8]$, whereas in the case of the lateral spring, only conventional techniques have been reported, e.g. by Neubauer et al., a round wire with iridium, tungsten and diamond tips [9]. For a micromachined cantilever, one with a square cross section would be ideal to realize equal spring constants in both directions.

This is no longer achievable with thin film cantilevers since the width of the beam should 
then become smaller than $1 \mu \mathrm{m}$, which would cause serious problems both in fabrication and measuring. In this paper, we are going to describe beams in monocrystalline silicon (m-Si) with a designed side length of the quadratic cross section of about $14 \mu \mathrm{m}$ and an integrated tip. Silicon is, by the way, very well suited for resonance methods because of its very high intrinsic $Q$-factor $[10]$.

\section{Fabrication of bidirectional cantilevers}

Theoretical studies have been carried out to find the shape and dimensioning according to the mechanical system requirements, i.e. a force constant $k<1 \mathrm{~N} / \mathrm{m}$ for an accurate force detection, a resonance frequency $\nu>10 \mathrm{kHz}$ to be able to filter vibrational noise out of the signal and a high force constant in $x$ direction. In order to find a compact shape with feasible dimensions, we investigated analytically a simple beam and different folded structures.

A meander-type cantilever allows us to shorten the overall length of the cantilever up to half of the length of the simple beam with the same cross section and force constant. Also, it is possible to adjust the ratio of the stiffness in $y$ and $z$ direction to a certain degree (from 1 for a simple beam to 1.5 for a meander with a maximum width similar to the protruding length), since one movement is rather bending and the other torsionally determined.

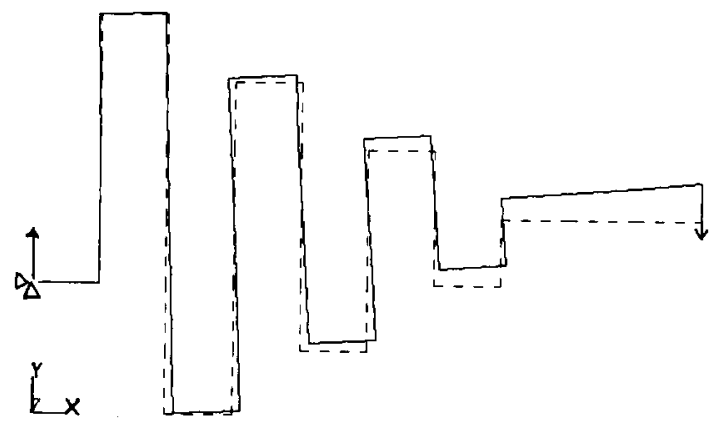

Fig. 1. The $y$ displacement of the meander cantilever simulated by ANSYS ${ }^{\odot}$.
Table 1

Mechanical characteristics of the meander cantilever (the beam width is $14 \mu \mathrm{m}$ and the protruding length $700 \mu \mathrm{m} ; k_{z}$ and $k_{y}$ are of the same order of magnitude, whereas $k_{x}$ is high)

\begin{tabular}{ll}
\hline $\begin{array}{l}\text { Force constant } \\
{[\mathrm{N} / \mathrm{m}]}\end{array}$ & $\begin{array}{l}\text { Resonance frequency } \\
{[\mathrm{kHz}]}\end{array}$ \\
\hline$k_{z}=0.79$ & $f_{z}=12$ \\
$k_{y}=1.02$ & $f_{y}=14$ \\
$k_{x}=20.4$ & $f_{x}=29$ \\
\hline
\end{tabular}

By the simulation program ANSYS ${ }^{\odot}$ [11] we optimized the shape (see fig. 1). Note that the clamping is not at the central line, which improves the decoupling of the $y$ movement from the $x$ movement. The results of the simulation of the optimized structure is listed in table 1. For a force at the end of the cantilever of $10 \mu \mathrm{N}$ we found that the maximal stress at the clamping $\left(\sigma_{\max }=15 \times 10^{6} \mathrm{~N} / \mathrm{m}^{2}\right)$ is about hundred times below the fracture stress of silicon.

On a double-side polished $280 \mu \mathrm{m}$ thick $\langle 100\rangle$ wafer, a $1.5 \mu \mathrm{m}$ thermal $\mathrm{SiO}_{2}$ was grown. On the back windows were opened for the formation of a membrane ( $\sim 15 \mu \mathrm{m}$ thick). This was obtained by anisotropic etching with $40 \% \mathrm{KOH}$ at $60^{\circ} \mathrm{C}$ from the backside while the topside was protected by a mechanical chuck. On this membrane the cantilever was patterned by means of $6.5 \mu \mathrm{m}$ thick photoresist (AZ 4562). Anisotropic reactive ion

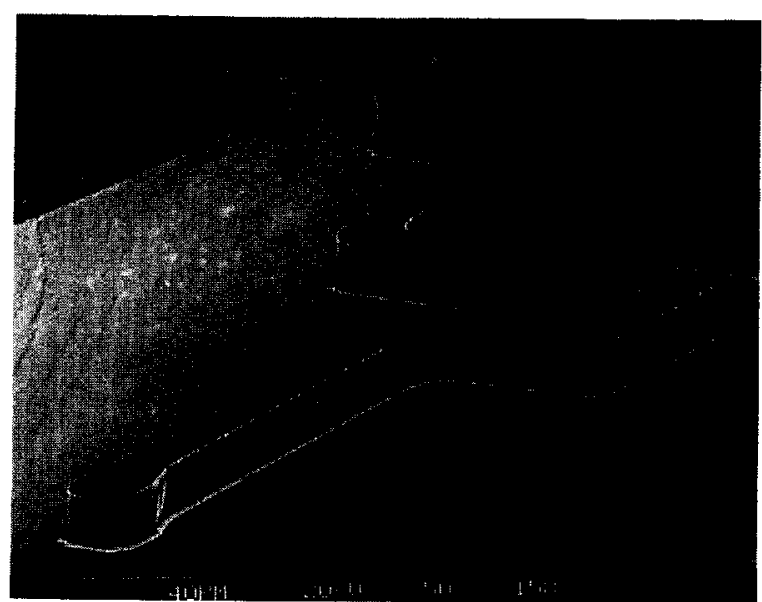

Fig. 2. m-Si cantilever with square cross section, designed as a meander shape. 
etching (RIE) using a $\mathrm{C}_{2} \mathrm{ClF}_{5} / \mathrm{SF}_{6}$ gas mixture ends in fairly vertical sidewalls [12]. We could thus fabricate cantilevers with a quadratic cross section (see fig. 2).

\section{Fabrication of tips on wafer}

A crucial part of the AFM point probe is the tip, which has to be as sharp as possible. It would be of great benefit to integrate it directly on the cantilever. In the field of vacuum microelectronics both wet and dry etching techniques to form sharp silicon field emitters were recently published [13], not all suitable for batch-processed integration on a cantilever, e.g. electron-beam deposition inside a SEM [14] or evaporation of a metal through an orifice [5].

Sharp Si tips can be achieved with a two-stage isotropic-anisotropic etching, exploiting the selfsharpening due to silicon crystal plane orientation [15], or using reactive ion etching techniques with dioxide or nitride etch masks $[5,16]$. We investigated methods to form tips in $\mathrm{m}-\mathrm{Si}$, which are compatible with the microfabrication of cantilevers.

We found that dry etching allows one to form sharper tips than wet etching. The best results, however, were obtained by a combination of dry and wet etching, which has been applied to form sharp tips with a high aspect ratio. By means of two successive photolithographies, we patterned a $10 \mu \mathrm{m}$ square double-layer mask consisting of 1.5

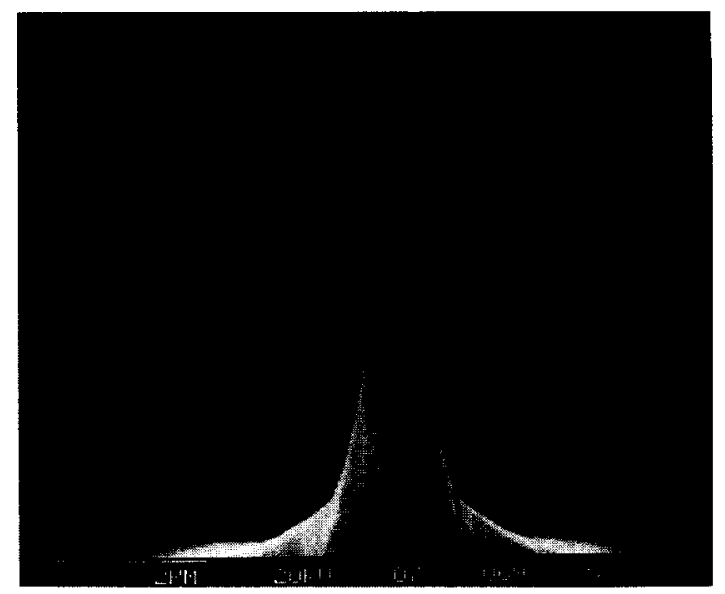

Fig. 4. A SEM of a $10 \mu \mathrm{m}$ high tip with a high aspect ratio.

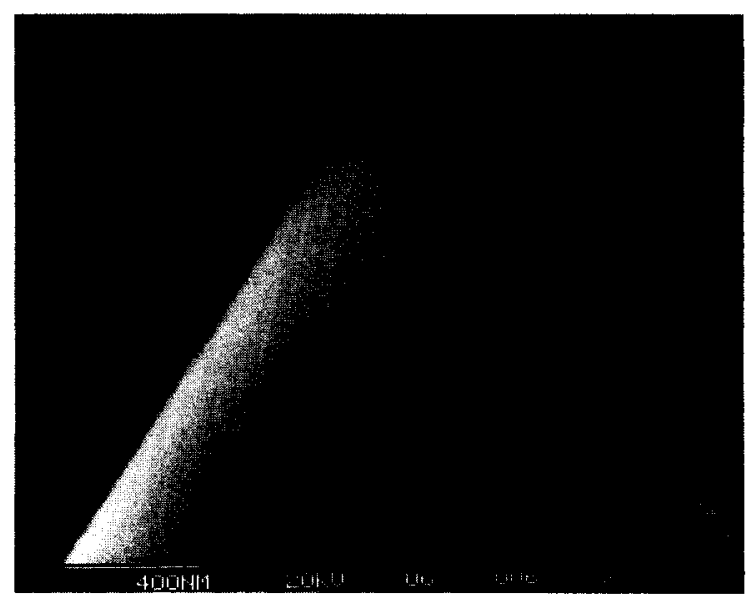

Fig. 5. The SEM of the end of the tip showing a tip radius of estimated $20 \mathrm{~nm}$.

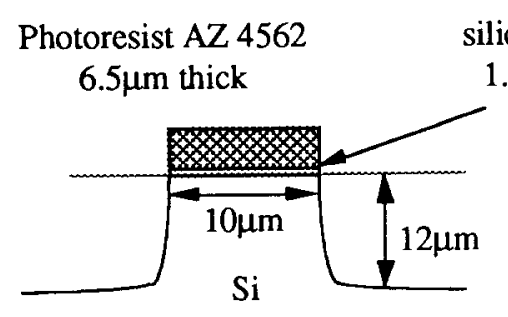

(a) ilicon dioxide

$1.5 \mu \mathrm{m}$ thick

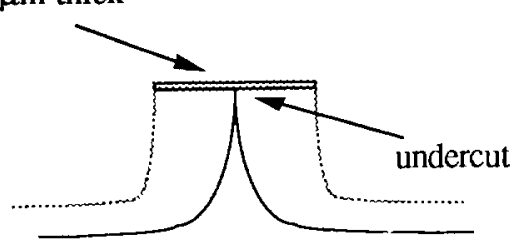

(b)

Fig. 3. Tip formation exploiting a two-stage dry-anisotropic/wet-isotropic etch process; (a) $12 \mu \mathrm{m}$ high silicon columns obtained by RIE etching, (b) column thinning and pronounced tip formation by wet etching. 
$\mu \mathrm{m}$ thick silicon dioxide (below) and $6.5 \mu \mathrm{m}$ thick photoresist AZ 4562 (above) as shown in fig. 3.

Application of the same RIE process as above results in silicon columns of $12 \mu \mathrm{m}$ height, with nearly vertical sidewalls and without under-cutting (fig. 3a). Subsequently the column is thinned during a $15 \mathrm{~s}$ isotropic acid etch step in $\mathrm{HNO}_{3}: \mathrm{HF}: \mathrm{CH}_{3} \mathrm{COOH}$. The silicon dioxide cap, previously buried under the photoresist layer, functions now as the etch mask and enhances the formation of a sharply pronounced tip as shown in fig. 4 . Note the high aspect ratio. A close-up is shown in fig. 5.

\section{Fabrication of tips on cantilever}

So far, Si cantilevers are published only for (one-directional) SPM applications without integrated tips $[17,18]$. IBM has announced such $\mathrm{m}-\mathrm{Si}$ cantilevers with an integrated tip, but without publicizing the fabrication process [19]. Albrecht [5] described some methods of micro-fabricating tips on silicon dioxide films.

By combination of the methods described in the sections above, we realized cantilevers with a quadratic cross section and an integrated tip. We exploited successive wet and dry etching techniques using appropriate masking materials as described now.

The formation of the cantilever was similar to that without tip, except that simultaneously with the opening of the windows on the backside for the machining of the membrane, at the topside 10 $\mu \mathrm{m}$ wide squares were patterned serving as mask for the subsequent tip formation. Moreover, the membrane was $25 \mu \mathrm{m}$ instead of $15 \mu \mathrm{m}$, since it will be etched further during the tip formation. The photoresist (AZ 4562) covers the $\mathrm{SiO}_{2}$ cap and serves as masking for the following RIE step (fig. 6a).

Again the same $\mathrm{C}_{2} \mathrm{ClF}_{5} / \mathrm{SF}_{6}$ gas mixture was used to etch the $15 \mu \mathrm{m}$ deep vertical sidewalls of the cantilever (see fig. 6b) and to obtain a very thin $(10 \mu \mathrm{m})$ membrane. Next an acid etching with a mixture of $\mathrm{HNO}_{3}: \mathrm{HF}: \mathrm{CH}_{3} \mathrm{COOH}$ is carried out during about $2 \mathrm{~min}$. This final etch step is crucial since simultaneously it forms the tip
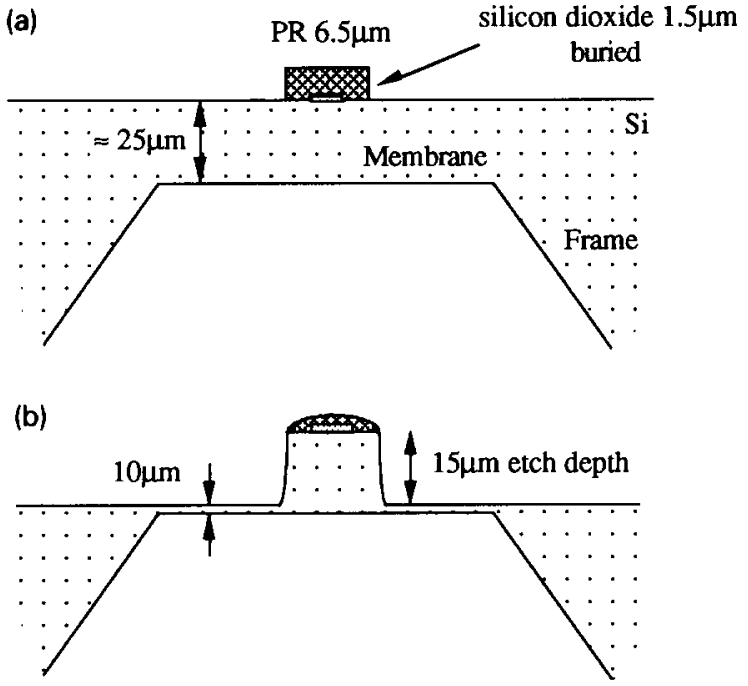

(c)
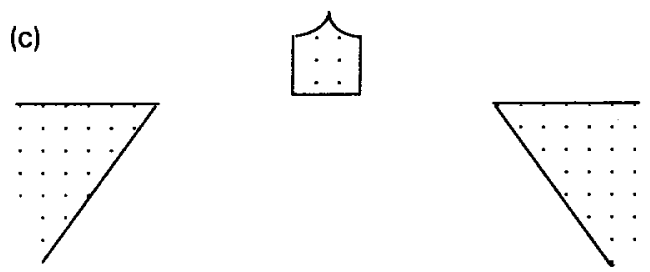

Fig. 6. Fabrication process for a silicon beam with integrated tip: (a) patterning of double-layer oxide-resist mask onto pre-processed $25 \mu \mathrm{m}$ thin membrane, (b) dry etching of rough cantilever shape until $10 \mu \mathrm{m}$ thin membrane, (c) final wet etching step releasing the spring, forming the tip and smoothing the surface.

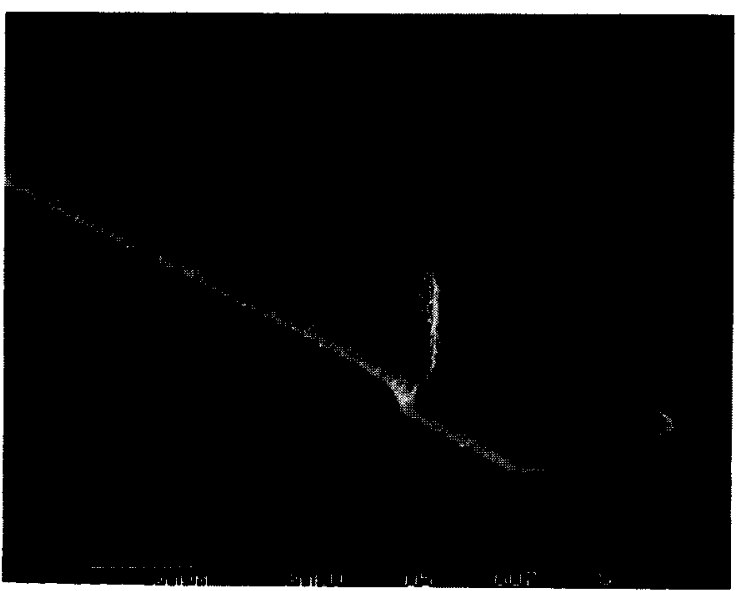

Fig. 7. Integrated $\mathrm{m}-\mathrm{Si}$ Tip on top of the cantilever. 
(using the buried oxide cap as mask), pierces through the membrane and consequently releases the silicon spring. Additionally, this wet etching smooths the dry-etched rough surface and cleans it from burned organic photoresist (figs. $6 \mathrm{c}$ and 7).

\section{Discussion}

The application of these cantilevers in force/friction measurements has to show whether folded or straight cantilevers are better suited and how they compare with the torsional method.

For the detection of the displacement we think of an optical method as discussed by Rugar [20], where two perpendicular optical beams are focussed on the cantilever, maybe with the combination of integrated optics elements, since we think that this approach has the greatest flexibility to use different cantilever and tip types. First measurements with the folded cantilevers as in normal AFM have been carried out with encouraging results.

In conclusion, we could show the realization of a monocrystalline silicon lever with an integrated silicon tip for a force/friction microscope. Moreover, sharp tips with a high aspect ratio could be demonstrated. Of course the described method can also be used to fabricate high-aspect-ratio cantilevers with an integrated tip, which is even easier than fabricating the tip on a square cross section.

\section{Acknowledgements}

We would like to thank Professor Güntherodt from the University of Basel and his co-workers for the support of this work and Mr. C. Ketterer for the SEM photographs.

\section{References}

[1] K. Wickramasinghe, J. Vac. Sci. Technol. A 8 (1990) 363.

[2] E. Meyer et al., J. Microscopy 152 (1988) 269.

[3] C.F. Quate, in: Proc. IEEE Micro Electromechanical Systems, Napa Valley, CA, 11-14 February 1990, p. 188.

[4] R.A. Buser, PhD Thesis, University of Neuchatel, June 1989.

[5] T.R. Albrecht, S. Akamine, T.E. Carver and C.F. Quate, J. Vac. Sci. Technol. A 8 (1990) 3386.

[6] L.C. Kong, B.G. Orr and K.D. Wise, in: Tech. Dig. IEEE Solid-State Sensor and Actuator Workshop, Hilton Head Island, South Carolina, 4-7 June 1990, p. 28.

[7] O. Marti, J. Colchero and J. Mlynek, Nanotechnology 1 (1990) 141.

[8] G. Meyer and N.M. Amer, J. Appl. Phys. Lett. 57 (1990) 2089.

[9] G. Neubauer, S. Cohen, G. McClelland, D. Horne and M. Mate, Rev. Sci. Instr. 61 (1990) 2296.

[10] R.A. Buser and N.F. de Rooij, Sensors Actuators A $21-23$ (1990) 323.

[11] ANSYS is a well known FEM program distributed by Swanson Analysis Systems, Inc., Houston.

[12] C. Linder, T. Tschan and N.F. de Rooij, Dry etching techniques as a new IC compatible tool for silicon micromachining, presented at Transducers 91.

[13] R.E. Turner, Ed., Proc. 2nd Int. Conf, on Vacuum Microelectronics, Bath, 24-26 July 1989, Inst. Phys. Conf. Ser. 99 (1989).

[14] Y. Akama, E. Nishimura, A. Sakai and H. Murakami, J. Vac. Sci. Technol. A 8 (1990) 429.

[15] P.C. Allen, in: ref. [13] pp. 17-20.

[16] J.B. Warren, in: ref. [13] pp. 37-40.

[17] T.W. Kenny, S.B. Waltman, J.K. Reynolds and W.J. Kaiser, in: Proc. IEEE Micro Electromechanical Systems, Napa Valley, CA, 11-14 February 1990, p. 192.

[18] J. Gimzewski and W. Pohl, IBM, European Patent Application Nr. 851022554.4, Date of publication 17.9.86, Bulletin $86 / 38$.

[19] O. Wolter, Th. Bayer, J. Greschner and H. Weiss, in: Tech. Dig. MME'90, 2nd Workshop on Micromachining, Micromechanics and Microsystems, Berlin, November 1990.

[20] D. Rugar, H.J. Mamin, R. Erlandsson, J.E. Stern and B.D. Terris, Rev. Sci. Instr. 59 (1988) 2337. 